\section{Enseñanza del Tiro Parabólico en la Universidad: Uso de simulaciones}

Recibido: 1 Agosto 2013 - Revisado: 30 Septiembre 2013

Aceptado: 30 Octubre 2013 - Publicado: 30 Diciembre 2013

Nilda Elena Lopez

Profesor Adjunto, Departamento de Física

Facultad de Ingeniería, Av. Libertador San Martín (Oeste) 1109 , San Juan, Argentina.

Ana Estela Puzzella

Profesor Asociado, Departamento de Física y de Química, Instituto de Investigación en de Filosofía. Humanidades y Artes, Av. José lgnacio de la Roza (Oeste) 230, San Juan, Argentina. anapuzzella@yahoo.com.ar

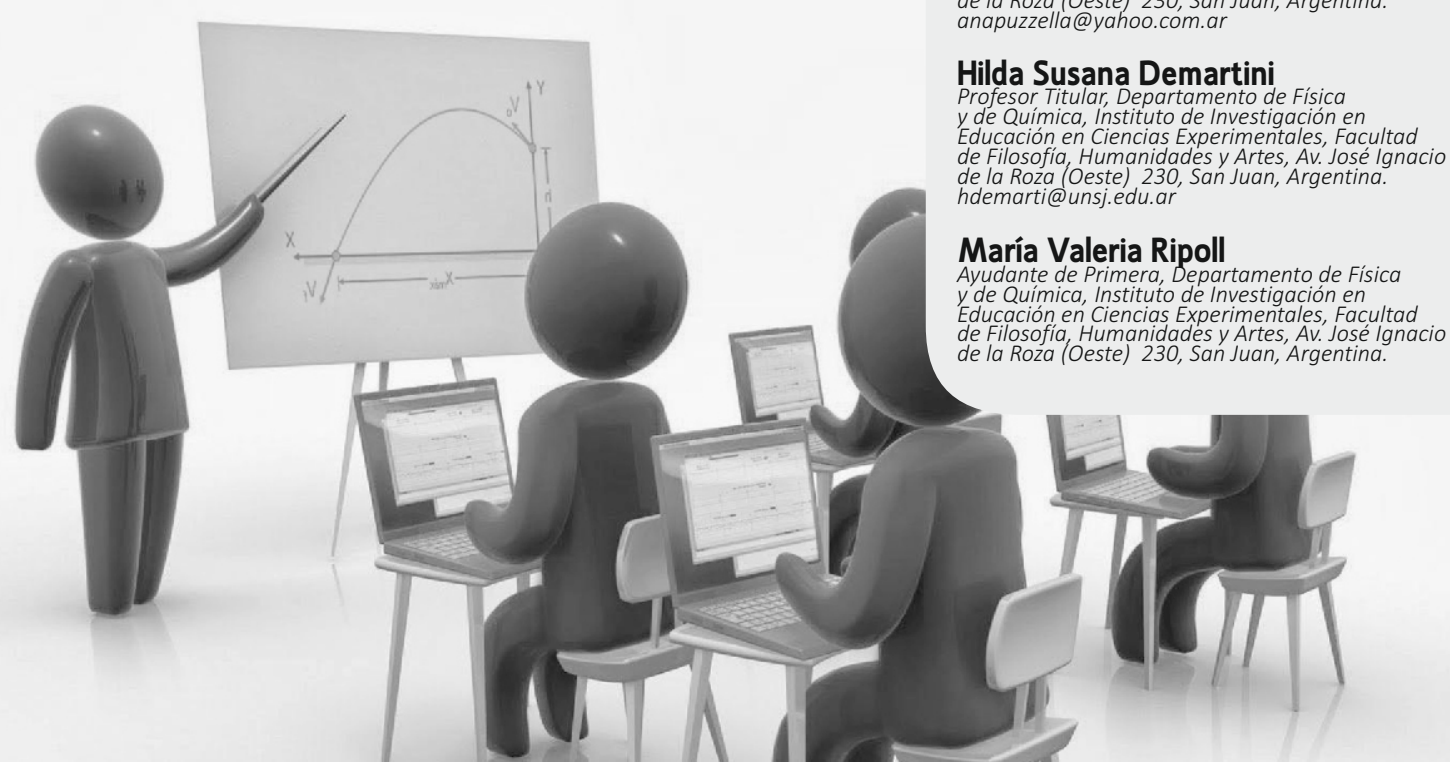

Resumen: En el presente trabajo se muestra los resultados de una experiencia con recursos educativos abiertos, simulaciones, sobre el tema Movimiento de Proyectiles. La misma se desarrolló con alumnos del Ciclo Básico de Ingeniería en Agrimensura y Electrónica y Profesorado en Química, que cursaban Física Mecánica. Para la ocasión, se trabajó con simulaciones y una guía de actividades (orientadora de la tarea). Se consideran tres momentos de comparación: momento tradicional, momento con simulación y aprendizaje autónomo y momento con simulación y la guía del docente. Los datos indicaron que los mejores resultados corresponden a la tercera instancia, es decir, que para los estudiantes de este nivel en el Ciclo Básico, sigue siendo importante el acompañamiento del profesor. No es significativo el aprendizaje desde el punto de vista tradicional, ni desde el punto de vista del autoaprendizaje sin la guía del docente.

Palabras clave: autoaprendizaje; ciclo básico de nivel universitario; movimiento de proyectiles; simulaciones.

Abstract: In the current work, we show the results from a study carried out with simulations designed to teach the subject of Projectile Motion. The study was performed on students enrolled in the course of Mechanics as part of the basic course work for the degrees of Surveying Engineer, Electronics Engineer and Chemistry Teacher. For the purpose of this study, we used simulations to compare learning in three moments: traditional moment, autonomous learning using simulations moment, and use of simulations and teacher's guidance moment. The results indicated that the best out come was produced by the use of simulations with the teacher guidance. This indicates that for students at the basic courses, it is still important to have supervision from a teacher. Learning in the traditional situation and during autonomous learning using simulations showed no significant difference. Key Words: self-learning, basic course work at university level, projectile motion, simulations. 


\section{INTRODUCCIÓN}

En la presente comunicación, se comentan los resultados del desarrollo de una actividad con simulaciones de Física, que se llevó a cabo con alumnos del Ciclo Básico universitario de carreras de Ingeniería y Profesorado en Química, donde la asignatura Física es una de las troncales de las carreras.

Asumiendo que el cambio vertiginoso y permanente que caracteriza a la sociedad actual está centrado, entre otros, en el desarrollo acelerado y permanente de las tecnologías de la información y comunicación (TIC), se acudió al uso de una simulación como complemento de las clases teóricas y prácticas. Actualmente, este recurso en los procesos de enseñanza-aprendizaje parece incuestionable $y$, por ello, se trabajó con un tema que presenta dificultades de comprensión, como es Tiro Parabólico.

Se supuso que las habilidades de los jóvenes en el manejo de recursos provenientes de la web, promoverían el aprendizaje autónomo. Partiendo de esta premisa, se propuso la tarea a realizar según una secuencia (que se explica en la Metodología), asumiendo que el aprendizaje sería logrado.

La experiencia ha dado muestras que, aunque los alumnos son mayores, no tienen las competencias para el autoaprendizaje con recursos de este tipo y aún necesitan de la guía del docente.

\section{ANTECEDENTES}

Se supone que los estudiantes de este siglo se sienten motivados y atraídos por las Tecnologías de Información y Comunicación (TIC). Sin embargo, también existen estudios que analizan el impacto que producen las TIC en los resultados del aprendizaje, e indican que no hay ninguna diferencia significativa entre los que pasaron por la experiencia informática y los que siguieron en la enseñanza tradicional
(Marchesi y Martín, 2003; cit.Tedesco, 2010 y González, 2004; Rodríguez et al., 2009).

Por otra parte, al efectuar una exploración en trabajos de investigación educativa en Física, se observa, entre otras cosas, que existe en los estudiantes un desinterés y una falta de motivación cada vez mayor por el aprendizaje de esta ciencia.

Desde hace algunos años, este grupo de docentes está realizando intentos de diseñar y aplicar estrategias conel propósito de promover dicho interés, la motivación y también el desarrollo de competencias específicas y transversales con alumnos de nivel medio y de los primeros años de la universidad. No obstante, aunque se notaron ciertas mejoras, se observan aspectos difíciles de revertir, tales como la integración grupal en el trabajo colaborativo y la autorregulación del aprendizaje, resultados que han sido comentados en comunicaciones anteriores (Lopez et al., 2007; (Puzzella et al., 2008; Lopez et al., 2009; Puzzella et al., 2012). Esto parece contradictorio debido a que la mayoría de los contenidos de Física son integradores y significativos por sí mismos, ya que admiten trabajar situaciones problemáticas en forma multidisciplinaria y contextualizada (Benito et al., 2010), además de la propia motivación que produce el uso de Internet en los adolecentes.

Para enfrentar este nuevo panorama y con el propósito desfavorecer el interés, la motivación y colaborar así con los estudiantes en el desarrollo de las competencias específicas y transversales, este grupo de trabajo implementó el uso de Recursos Educativos Abiertos (REA), en particular las denominadas Simulaciones Informáticas.

Dichas simulaciones son interactivas y crean situaciones artificiales, las cuales permiten visualizar fenómenos que se acercan en alguna medida a la situación real y proporciona una mejor comprensión de conceptos que, por su complejidad, constituyen una tarea difícil para los alumnos (Cabrero et al., 2003). 
En particular, en esta experiencia, se utilizaron las simulaciones diseñadas en un proyecto del grupo de investigación en educación en la Física de la Universidad de Colorado (PhETs, 2011) y las del Curso de Física con Ordenador de Ángel Franco García (2011).

\section{METODOLOGÍA}

Se trabaja con una metodología activa, para indagar la incidencia del uso de recursos educativos abiertos (REA) en clases de Física. Se trata de promover la observación, interpretación, uso y rehúso de estos recursos para el aprendizaje del Movimiento de Proyectiles.

\subsection{Muestra}

Se trabaja con 124 alumnos de un curso de Física Básica de Ingeniería en Agrimensura y Electrónica y 20 del Profesorado en Química, de la Universidad Nacional de San Juan.

\subsection{Tema y recursos seleccionados}

El tema seleccionado obedece, entre otras razones, a: (a) los resultados de la evaluación, luego de las clases tradicionales, no es satisfactoria; (b) existen simulaciones muy completas e interactivas para su estudio, que podrían ayudar al aprendizaje.

En la actividad se optó por utilizar los recursos educativos abiertos propuestos por García (2011)(Phet, 2011), que son sencillas de ejecutar pero potentes para aclarar los conceptos involucrados.

\subsection{Instrumento de evaluación}

La evaluación de los alumnos se lleva a cabo en diferentes instancias, teniendo carácter de Pretest y/o Postest, según corresponda. En todos los casos se toma la misma evaluación (una prueba de lápiz y papel) en la cual deben responder a situaciones del Movimiento de Proyectiles. En ella, los estudiantes deberían tener claridad acerca de las magnitudes que intervienen y su naturaleza (escalar o vectorial). La prueba tiene ocho ítems, identificados desde a hasta h.

\subsection{Desarrollo}

\subsubsection{Primera etapa: Clase tradicional}

Se desarrolló el tema Movimiento de Proyectiles, en forma tradicional. Se observaron dificultades en la interpretación de las magnitudes involucradas, sobre todo en el carácter vectorial, lo que se ha puesto de manifiesto en la evaluación con una prueba (de lápiz y papel) que tiene carácter de Postest para esta instancia y Pretest, para la posterior. Los resultados se muestran en PosT-PreSA (Análisis de los datos).

3.4.2 Segunda etapa: Uso de simulación sin intervención docente

Con el propósito de revisar y mejorar el aprendizaje del tema, se invitó a los estudiantes a que trabajaran las simulaciones propuestas. Para llevar a cabo la actividad, se proporcionó a los alumnos, una guía para que ordenaran su tarea. Esta instancia se realizó bajo el supuesto que el estudiante universitario es consciente de su compromiso con el proceso y, por tanto, es capaz de generar sus propios aprendizajes; es decir, se intentó potenciar su autoaprendizaje a través del juego de la simulación.

Posteriormente, se los evaluó con la misma prueba de la etapa anterior que tuvo carácter de Postest en esta instancia y de Pretest, para la siguiente etapa. Los resultados se muestran en PosSA-PreAy (Análisis de los Datos).

3.4.3 Segunda etapa: Uso de simulación sin intervención docente

Debido a que los resultados no fueron los esperados, se retoma la simulación en clase con la guía de trabajo y la orientación de los docentes del curso, para rescatar los aspectos relevantes de la simulación. Se observó una mejora en los aspectos conceptuales de las magnitudes vectoriales involucradas en el tema. Los resultados se muestran en PosAy (Análisis de los Datos). 


\section{ANÁLISIS DE DATOS}

En este apartado, sólo se muestran los resultados de las respuestas a algunas preguntas, que fueron las que presentaron mayor confusión conceptual. La Fig. 1 corresponde a las preguntas que estaban referidas a dos trayectorias de una pelota en diferentes condiciones iniciales de lanzamiento.

Pregunta b: "Una vez que la pelota abandona la mano del lanzador, ¿actúa alguna aceleración? ¿Cuál?”. En general, identificaron correctamente que actúa en todo instante la aceleración de la gravedad.

a)

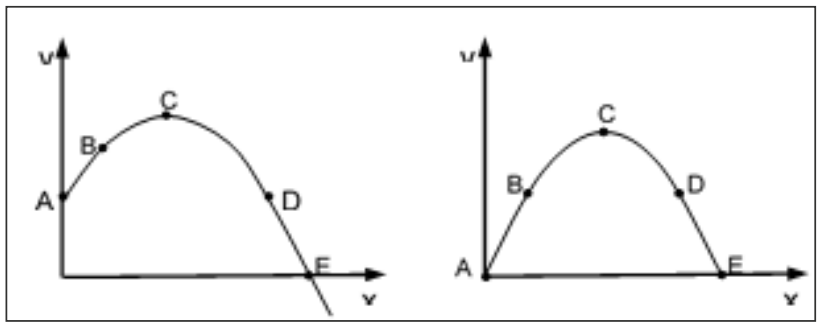

Figura 1: Gráficas de la trayectoria de dos pelotas lanzadas con la misma velocidad inicial. En (a) tiene una posición inicial de lanzamiento. En (b) la posición inicial es cero.

Pregunta c: Se solicitó dibujar los vectores velocidad y aceleración en cada uno de los puntos indicados, respetando la magnitud relativa de cada vector. Solo se muestran en la Fig. 2, los resultados para la velocidad y aceleración de la Fig. 1a; los porcentajes son similares para la otra situación.

En la categoría MB se considera que dibuja el vector en la dirección y sentido correctos, dibuja componentes $\mathrm{y}$, además, lo hace a escala. En la B dibuja los vectores pero no respeta la escala. En $\mathrm{R}$, dibuja únicamente el vector resultante y no respeta la escala.

Se mostró que la interpretación vectorial de las magnitudes mejoró a medida que se desarrolla la simulación con la ayuda del docente. Después de la enseñanza tradicional, no se logró una interpretación correcta y aunque hubo un avance con la simulación trabajada en forma autónoma, se evidenció una falta de observación de los vectores que aparecían dibujados, con distintos colores y cambiando (o no) su magnitud y dirección, según corresponda.

Pregunta: Se comparó la aceleración en el punto de altura máxima (C) con la de la posición de lanzamiento (A). La respuesta correcta es igual, en ambas situaciones. Se observó que aumentaron los porcentajes de la respuesta correcta; no obstante, luego de trabajar la simulación (PosSA), un 49\% continuó respondiendo incorrectamente, porcentaje que mejoró al 87,5\% de respuestas correctas (PosAy, Fig. 3).

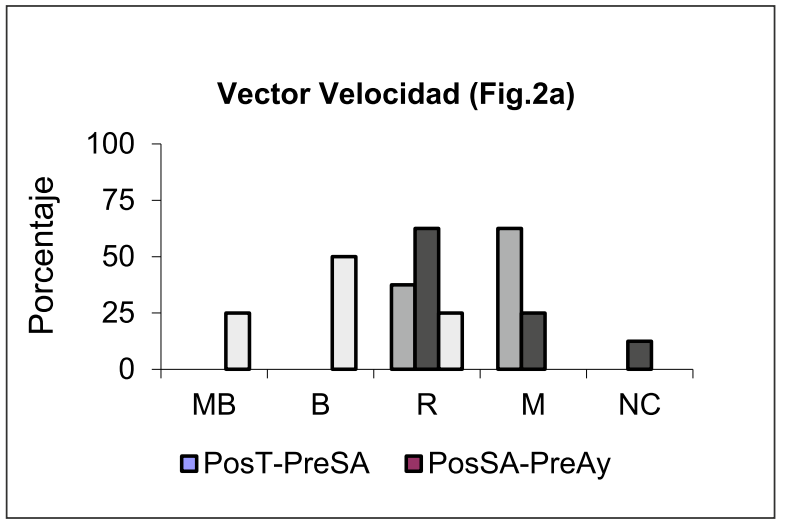

Figura 2. Respuestas para los vectores velocidad y aceleración de la Fig. 2.a del test.

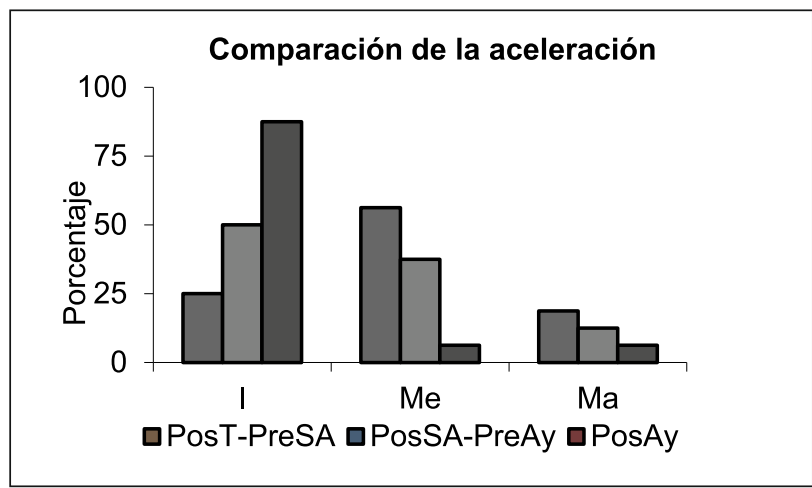

Figura 3: Resultados del las respuestas que comparan la aceleración en las diferentes puntos del las trayectorias. 
Pregunta f: Se solicitó comparar la rapidez en el punto D con la rapidez en A, de los esquemas de la Fig.1. Como puede apreciarse, el punto $D$ es el simétrico del inicial de lanzamiento, en la Fig. 1a, por lo que la respuesta correcta, para este caso, es Igual (“I"). Se observó que con el autoaprendizaje, disminuye el porcentaje de respuestas correctas (de casi el 60\% en el Pretestal $50 \%$ en el Postest) y al trabajar la simulación con el docente, el porcentaje anterior aumentó al 87,5\%.

En cuanto a la Fig.1b, el punto $D$ no es el simétrico, sino que está a mitad de camino, aproximadamente, entre la altura máxima y el punto donde cae al suelo, por lo que la respuesta correcta es Menor ("Me"). Con la simulación, mejoró la interpretación conceptual de la rapidez, nuevamente con el acompañamiento del docente para interpretar. Los porcentajes de respuestas se muestran en la Fig. 4.

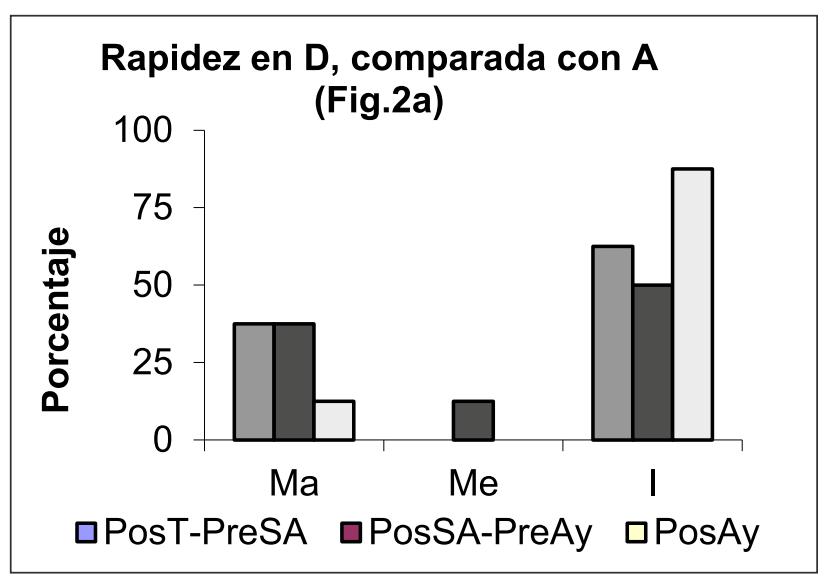

Figura 4: Comparación de la rapidez en dos puntos de la trayectoria, para las figuras $2 a$ y $2 b$ del test.

Pregunta g: Se pide comparar la rapidez en el punto de llegada (E) con respecto al punto de lanzamiento inicial (A). La simulación con aprendizaje autónomo produjo una mejora no significativa en los resultados en ambos casos.

\section{CONCLUSIONES}

En general, de los resultados de esta experiencia, se observaron dos aspectos principales:

a) Una falta de compromiso de los estudiantes universitarios con el proceso de aprendizaje cuando se trata de la autogeneración de los conceptos. El análisis de los datos pondría de manifiesto una actitud pasiva y poco observadora en lo referido al trabajo con simulaciones, aún cuando se supone que los jóvenes se motivan con el uso de las TIC.

b) Los estudiantes necesitaron la orientación constante del docente para la interpretación de los parámetros que ofrecen las simulaciones, tal como se comenta en los análisis de los tests.

Por lo tanto, los resultados de este trabajo dieron un indicio que la inclusión de estrategias usando como recurso las simulaciones en la universidad, debe ser atendida por el docente, quien no debe resignar su responsabilidad como orientador del proceso y las actividades deberían ser convenientemente programadas y acompañadas, aún cuando se trate de alumnos que se encuentran cursando estudios superiores. En las circunstancias mencionadas, el uso de simulaciones en el proceso de enseñanza y aprendizaje de Física puede ser un buen complemento, pero no deberían sustituir completamente a otras actividades. 


\section{REFERENCIAS}

Benito, A, Portela, A. y Jiménez, M. (2006). Análisis de la enseñanza de la Física en Europa: el fomento de competencias generales en estudiantes universitarios [versión electrónica]. Revista Iberoamericana de Educación, 38(7), pp.1-5. Recuperado de

http://www.oei.es/oeivirt/enfisica.htm.

Cabero, J., Castaño, C., Cebreiro, B., Gisbert, M., Martinez, F., Morales, J.A., Prendes, M.P., Romero, R. y Salinas, J. (2003). Las nuevas tecnologías en la actividad universitaria. Píxel-Bit 20. Recuperado de: http://www.sav.us.es/pixelbit/pixelbit/articulos/n20/ n20art/art2008.htm

Franco García, A. (2011). Movimiento bajo la aceleración constante de la gravedad. Física con O r d e n a d or. R e c u p e r a d o d e : http://www.sc.ehu.es/sbweb/fisica/cinematica/para bolico/parabolico.htm

González, Pérez, A. (2004). Reseña de Tecnología y aprendizaje. Investigación sobre el impacto del ordenador en el aula. Biblios,5(19). Recuperado de http://www.redalyc.org/articulo.oa?id=16101905.

Lopez, N., Puzzella, A., Alborch, A., Zabala, A., Demartini, H. y Cabrera, L. (2007). Profile of prospective bioengineering students at National University of San Juan.J. Phys.: Conf. Ser. 90 [012016], p. 8.Doi: 10.1088/1742-6596/90/1/012016.

Lopez, N., Puzzella, A. y Alborch, A. (2009). Webquest: una estrategia interesante para la enseñanza de la Física en el nivel secundario. X Encuentro Internacional Virtual Educa. Recuperado de www.virtualeduca.info/ponencias2009/566/LOPEZP UZZELLAALBORCH(VirtualEduca).doc

Marchesi, A. y Martín, E.(2003). Tecnología y aprendizaje; Investigación sobre el impacto del ordenador en el aula. Madrid: Instituto IDEA.
Puzzella, A., Lopez, N., Alborch, A., Demartini, H., Zabala, A. y Cabrera, L. (2008).Los ingresantes a Ingeniería ¿Han desarrollado las competencias básicas necesarias para un buen desempeño académico? Resultados de una indagación. VI Congreso Argentino de Enseñanza de la Ingeniería. "Formando al Ingeniero del Siglo XXI". Buenos Aires: Universidad Nacional de Salta.

Puzzella, A., Quiroga, M.,y Lopez, N. (2012). Simulaciones como recurso para el aprendizaje del Movimiento de Proyectiles. ¿Qué competencias es posible Promover? 7 mas. JUCEN. Buenos Aires: Universidad Nacional de Catamarca.

Tedesco, J. C. (2010).Educación y justicia: el sentido de la educación. Documento Básico de la XXV Semana Monográfica de la Educación La educación en el horizonte 2020. Madrid: Fundación Santillana.. Disponible en:

http://www.fundacionsantillana.com/upload/fichero s/noticias/201011/documento_bsico.pdf.

University of Colorado at Boulder. PhetTM (2011).Interactive Simulations. Creative Commons. Recuperado de:

http://phet.colorado.edu/es/simulation/projectilemotion 